\title{
Special Issue on Applied Computational Science
}

\section{and Engineering}

\author{
Phaneendra K. Yalavarthy ${ }^{*}$
}

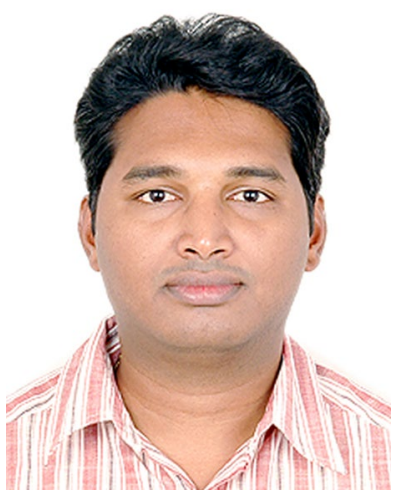

Guest Editor Editorial,

The special issue on applied computational science and engineering from the Journal of the Indian Institute of Science is a very timely issue as it reflects the paradigm shift towards accepting computational science as an important academic discipline. Even in traditional institutes like Indian Institute of Science (IISc), creation of a full-fledged academic department in computational science can be considered as a welcoming and important step.

The computational science and engineering includes elements of applied mathematics, computer science, and integrates them with domain knowledge of engineering and sciences. In addition, lately it has become indispensable for leading scientific and engineering investigations in number of industrial sectors, including aerospace, medical, biological, chemical, and material sciences. It complements theory and experiment to accelerate the scientific discovery. Having natural flavor of being interdisciplinary, the computational science and engineering was able to effectively analyze the complex systems and/or optimize the various elements associated with it.

At the forefront, the computational science and engineering stems from physical applications and this special issue has very selective five such reviews. As biomedical science is the biggest benefactor of computational science, there are reviews present on heart-rate estimation during physical exercise, which are highly non-linear signals to analyze. The multi-tissue health models of health and disease were also presented as a review of computational models of existing as well as futuristic expansions of inference methods for human genomic datasets. Computational methods to study fracture as well as differential systems were also presented in this special issue. The denoising methods for improving magnetic resonance diffusion images were also reviewed in this special issue. Overall, this issue presents five selective reviews of computational methods being applied to fields such as computational mechanics, controls systems, bioinformatics, signal, and image processing. Hope the readers also find it interesting.

Many thanks to Prof. Guru Row for giving this opportunity to edit this special issue and special thanks to Ms. Kavitha and Springer India team for all their help.
Department of Computational and Data Sciences, Indian Institute of Science, Bangalore 560 012, India ‘phani@cds.iisc.ac.in 